\title{
Measurement of the Spatial Evolution of the Deprotection Reaction Front with Nanometer Resolution using Neutron Reflectometry
}

\author{
Eric K. Lin ${ }^{1}$, Christopher L. Soles ${ }^{1}$, Dario L. Goldfarb ${ }^{2}$, Brian C. Trinque ${ }^{3}$, Sean D. Burns ${ }^{3}$, \\ Ronald L. Jones ${ }^{1}$, Joseph L. Lenhart ${ }^{1}$, Marie Angelopoulos ${ }^{2}$, \\ C. Grant Willson ${ }^{3}$, and Sushil K. Satija ${ }^{1 \mathrm{a}}$, Wen-li Wu ${ }^{1}$ \\ ${ }^{1}$ Polymers Division and ${ }^{1 a}$ Center for Neutron Research, National Institute of Standards and \\ Technology, Gaithersburg, MD 20899-8541. \\ ${ }^{2}$ IBM T. J. Watson Research Center, Yorktown Heights, NY 10598. \\ ${ }^{3}$ Departments of Chemistry and Chemical Engineering, University of Texas at Austin, Austin, TX \\ 78712.
}

\begin{abstract}
The use of chemically amplified photoresists for the fabrication of sub-100 $\mathrm{nm}$ features will require spatial control with nanometer level resolution. To reach this goal, a detailed understanding of the complex reaction-diffusion mechanisms at these length scales is needed and will require high spatial resolution measurements. In particular, few experimental methods can directly measure the spatial evolution of the deprotection reaction front and correlate it with the developed structure. In this work, we demonstrate the complementary use of neutron (NR) and x-ray (XR) reflectometry to measure the reaction front profile with nanometer resolution. Using a bilayer geometry with a lower deuteriumsubstituted poly(tert-butoxycarboxystyrene) (d-PBOCSt) layer and an upper poly(hydroxystyrene) (PHOSt) layer loaded with a photoacid generator (PAG), we directly measure the spatial evolution of the reaction front. We show that the reaction front profile is broader than the initial interface after a post-exposure bake and the compositional profile changes upon development in an aqueous base solution. We also directly correlate the final developed structure with the reaction front profile. The spatial detail enabled by this general methodology can be used to differentiate between and evaluate quantitatively reaction-diffusion models.
\end{abstract}

\section{INTRODUCTION}

The successful development of chemically amplified photoresists for the fabrication of sub-100 $\mathrm{nm}$ features will require nanometer scale spatial control of the deprotection reaction front. There are many challenges toward meeting this goal because of the difficulties in measuring and understanding correlations between processing conditions and critical dimension control at these length scales. Detailed information about the physics and chemistry controlling the local deprotection reaction will be required. In addition, physical quantities from larger length scale, bulk measurements may not be applicable for the fabrication of smaller structures. For example, ultrathin photoresist imaging layers will be required because of the increased optical absorption of the photoresist at smaller imaging wavelengths. There are reported changes in the material properties of ultrathin imaging layers such as the glass transition temperature, $\mathrm{Tg}$, or interfacial effects such as interdiffusion in a bilayer photoresist that can affect the fabrication process [1-3]. Direct measurement of the reaction front in ultrathin photoresist systems would be needed.

It is well established that the spatial evolution of the deprotection reaction front is controlled by many factors including the distribution of the amount of photogenerated acid proportional to the local DUV dose and acid diffusion during the post-exposure bake (PEB). There have been significant efforts toward quantifying acid diffusion rates and determining their effect on the final structure. Experimental methods have included the use of ion conductivity measurements $[4,5]$, correlation with analysis of the final structure [6], Fourier transform infrared spectroscopy (FTIR) 
measurements in varying sample geometries [7, 8], ellipsometry [9], thickness changes after reaction and development [1], and scanning electron microscopy on cleaved, chemically decorated structures [10]. These experimental methods have been limited by either spatial resolutions that are significantly larger than the nanometer length scales required at future technology nodes or the lack of direct information of the spatial evolution of the deprotection reaction front. Fundamental understanding and quantification of the reaction-diffusion process at these reduced dimensions will be needed to control, develop, and model the lithographic process.

Many models have been developed to describe the reaction-diffusion process in chemically amplified photoresists. Differences between the models largely lie in the description of the acid diffusion process and the detail in which the reaction kinetics are incorporated. Predictions from reaction-diffusion models have been compared with the total extent of reaction with FTIR data before development in several systems [7, 8, 12-14]. Top loss deprotection experiments and comparison with different acid diffusion descriptions showed that Fickian diffusion cannot explain the experimental results [11]. Further, it was suggested that the diffusion constant is a function of the deprotection fraction. Experiments with an acid feeder layer coupled to a deprotected polymer layer show fast reaction rates at short times (less than typical PEB times) then saturation at longer times [7, 10]. A single acid diffusion constant also cannot explain the data. As a result, it was proposed that the mechanism for reaction front propagation includes an enhancement of the local acid mobility at short times due to the evolution of deprotection reaction products. It was postulated that the volatile reaction products increase the local free volume to allow increased acid mobility. Additionally, the acid is subsequently trapped in the deprotected polymer matrix leading to a net reduction in the available acid for deprotection. Numerical calculations based upon this model show that the reaction front propagates as a sharply defined interface into the protected polymer layer [14]. Other successful modeling approaches include the use of a simulation incorporating in detail the deprotection reaction kinetics as well as diffusion mechanisms. These calculations show that FTIR data may be fit with a physical picture where Fickian acid diffusion coefficients are significantly different within the protected and deprotected polymer environments [8].

There is difficulty in clearly distinguishing between reaction-diffusion models because of the lack of a direct measurement of the reaction front before development or without any further sample preparation. Progress has been made by created contrast for SEM measurements by chemically decorating deprotected polymers with the silylation of samples cleaved normal to a diffusion-couple sample [10]. These experiments enable estimates of the spatial extent of the deprotection front and suggest that the reaction front propagates as a sharply defined band. However, the details of the silylation chemistry and subsequent etch step are not known. Further, although there are general guidelines for the composition of the solubility switch and simulation models for development effects on LER, there has been no direct correlation between the reaction front, its width, shape, and composition, and the final developed image. As development steps may also be sources of line-edge roughness, this connection is critical.

In this work, we present the first direct measurements of the reaction front with nanometer resolution both after PEB and after development using neutron reflectometry. Compositional and density depth profiles are measured from a well-defined bilayer structure with a lower layer consisting of the protected polymer and an upper layer consisting of the deprotected polymer loaded with the photoacid generator (PAG). The protected polymer is synthesized with deuteriumlabeled protection groups providing strong neutron scattering contrast. The neutron scattering contrast is then proportional to the local extent of reaction. X-ray reflectometry is used to measure the density profile of the bilayer structure enabling a direct comparison of the developed film structure with the spatial extent of the reaction front.

\section{EXPERIMENTAL}

Bilayers for the reflectivity measurements were prepared on $<111>$ silicon substrates $(3 \mathrm{~mm}$ thick, $75 \mathrm{~mm}$ diameter) primed with hexamethyldisilazane (HMDS). The lower layer consisted of poly(tert-butoxycarboxystyrene) (d-PBOCSt) $\left(\mathrm{M}_{\mathrm{r}, \mathrm{n}}=21000, \mathrm{M}_{\mathrm{r}, \mathrm{w}} / \mathrm{M}_{\mathrm{r}, \mathrm{n}}=2.1\right)$, the protected polymer, with a deuterium-substituted protection group. The upper layer consisted of poly(hydroxystyrene) (PHOSt) $\left(\mathrm{M}_{\mathrm{r}, \mathrm{n}}=5260, \mathrm{M}_{\mathrm{r}, \mathrm{w}} / \mathrm{M}_{\mathrm{r}, \mathrm{n}}=1.12\right)$, the deprotected polymer, loaded with a $5 \%$ mass fraction of the photoacid generator (PAG) di(tert-butylphenyl) iodonium perfluorooctanesulfonate (PFOS). Bilayers were also prepared with PHOSt without PFOS as control experiments. The deuterium-substituted ptert-butoxycarboxystyrene (d-PBOCSt) was synthesized using the following procedure. First, 2-methyl-2-propanol- $\mathrm{d}_{10}$ was reacted with 1 equivalent potassium metal in dry tetrahydrofuran (THF) to form potassium tert-butoxide- $\mathrm{d}_{9}$. The reaction was considered to be complete when all of the potassium appeared to dissolve in the THF; this generally took about $48 \mathrm{~h}$ at reflux temperature. This salt was then used as starting material for the published literature procedure for 
di-tert-butyl dicarbonate [15]. 1.1 Equivalents of di-tert-butyl dicarbonate- $\mathrm{d}_{18}$ were reacted with PHOSt with 0.1 equivalents 4-(dimethylamino)pyridine in dry THF. Precipitation into hexanes afforded a $95 \%$ yield of a white polymer that was $92 \%$ protected as measured by thermal gravimetric analysis. Control measurements comparing the deprotection kinetics by FTIR of exposed blanket films of d-PBOCSt and similarly prepared hydrogenous PBOCSt loaded with PAG show little difference between the two polymers.

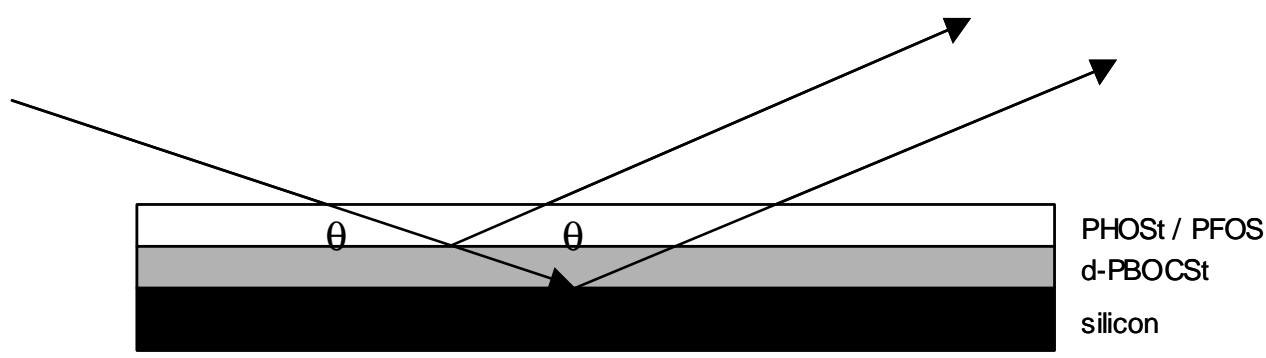

Figure 1. Schematic diagram of the bilayer structure and the reflectivity geometry for both neutrons and $\mathrm{x}$-rays. The deuterium-substituted PBOCSt layer is spin-coated on the silicon substrate. The PHOSt layer with and without PFOS is spin-coated directly onto the PBOCSt layer. The incident radiation is brought in a glancing angle, $\theta$.

The lower PBOCSt layer was spin-coated onto the silicon wafer from a propylene glycol methyl ether acetate (PGMEA) solution and post-apply baked (PAB) for $90 \mathrm{~s}$ at $130^{\circ} \mathrm{C}$. The upper PHOSt with PFOS layer was then spincoated directly onto the PBOCSt layer from a 1-butanol solution and again PAB for $90 \mathrm{~s}$ at $110^{\circ} \mathrm{C}$. The bilayer was exposed to broadband UV dose of $1000 \mathrm{~mJ} / \mathrm{cm}^{2}$. Next, the bilayer was post-exposure baked (PEB) for $30 \mathrm{~s}$ at $110^{\circ} \mathrm{C}$. Finally, the bilayer was developed by immersion in $0.26 \mathrm{~N}$ tetramethylammonium hydroxide (TMAH) for $30 \mathrm{~s}$ followed by a rinse in ultrapure water. X-ray and neutron reflectivity measurements were performed after each processing step. Separate identically prepared samples to minimize delay effects. For the data presented here, the developed bilayer samples were the same samples measured after PEB. For the hotplates and relatively thick silicon wafers used in this study, the surface of the silicon wafers reached a steady state temperature after approximately $60 \mathrm{~s}$. The steady state temperature was generally $(5 \text { to } 10)^{\circ} \mathrm{C}$ lower than the set point temperature.

Figure 1 shows a schematic diagram of the reflectivity geometry used to characterize the bilayers. The x-ray or neutron beams are sent at grazing incident angles, $\theta_{\mathrm{i}}$, to the sample surface and detected at the specular condition where the detection angle, $\theta_{\mathrm{r}}$, is equal to $\theta_{\mathrm{i}}$. Neutron reflectivity measurements were performed on the NG7 reflectometer at the National Institute of Standards and Technology Center for Neutron Research. The wavelength, $\lambda$, of the incident neutrons was $4.75 \AA$ with a wavelength spread, $\Delta \lambda \lambda=0.2$. X-ray reflectivity measurements were performed on modified x-ray diffractometers using a tube source with a wavelength, $\lambda$, was $1.54 \AA$ and wavelength spread, $\Delta \lambda / \lambda=0.1$. The data are plotted as the logarithm of the reflectivity (the ratio of the reflected intensity to the incident intensity) versus $q(q=(4 \pi / \lambda) \sin \theta)$, the momentum transfer of the neutrons or x-rays normal to the sample surface. In this configuration, the reflectivity data provide planar-averaged depth profiles. The reflectivity data were modeled using nonlinear least-squares fits with a recursive multilayer method [16]. Model real space depth profiles of the x-ray or neutron scattering length density are generated. When possible, the interfacial transition between layers is modeled with an error function. Then, the reflectivity is calculated and compared with the experimental data. Model parameters such as the polymer layer thickness or interfacial width were varied to minimize the difference between the model calculation and the reflectivity data. The reflectivity data were taken under ambient atmospheric conditions. All data in the paper and in the figures are presented with the standard uncertainty of the measurement.

The use of both x-ray and neutron reflectometry allows the determination of both the compositional and density depth profiles of the bilayer structure. The source of the scattering contrast of the bilayer material to $\mathrm{x}$-ray and neutron radiation is different. X-rays are scattered by differences in electron density whereas neutrons are scattered through interactions with the nuclei. The scattering contrast is quantified by the elastic coherent scattering length density, $\mathrm{Q}_{\mathrm{c}}{ }^{2}$, and is proportional to the composition and mass density of each component. There is a large and convenient difference between the scattering cross-sections of deuterium and hydrogen for neutrons [17]. Neutron contrast may be obtained 
through the substitution of hydrogen with deuterium in organic materials without significantly altering the chemistry of the system. The deuterium is placed only on the protection group of the PBOCSt polymer. In general, the acidcatalyzed deprotection reaction in this system can be written

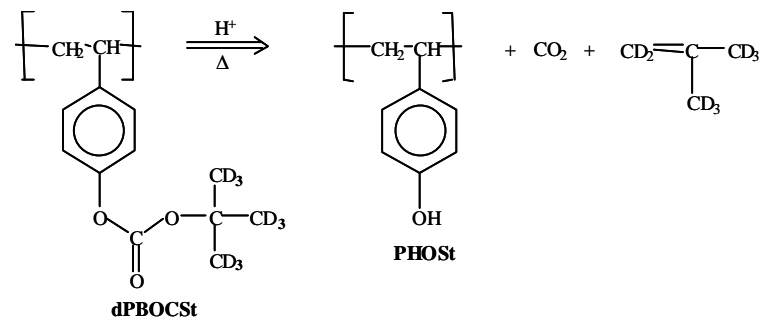

where the protection group of PBOCSt is cleaved leaving PHOSt and volatile reaction products including carbon dioxide and isobutylene $[18,19]$. The details of the reaction have been shown to be more complex with additional volatile reaction products and possible side-reactions [20]. However, the large majority of the reaction products involving the deuterium-substituted group are volatile and are released from the sample [21, 22]. The result is a large change in contrast for neutron reflectivity from the deuterium labeled PBOCSt polymer to the fully hydrogenous PHOSt product. PBOCSt and PHOSt have similar $\mathrm{x}$-ray scattering contrast, so the overall density profile of the bilayer structure is measured with $\mathrm{x}$-ray reflectivity.
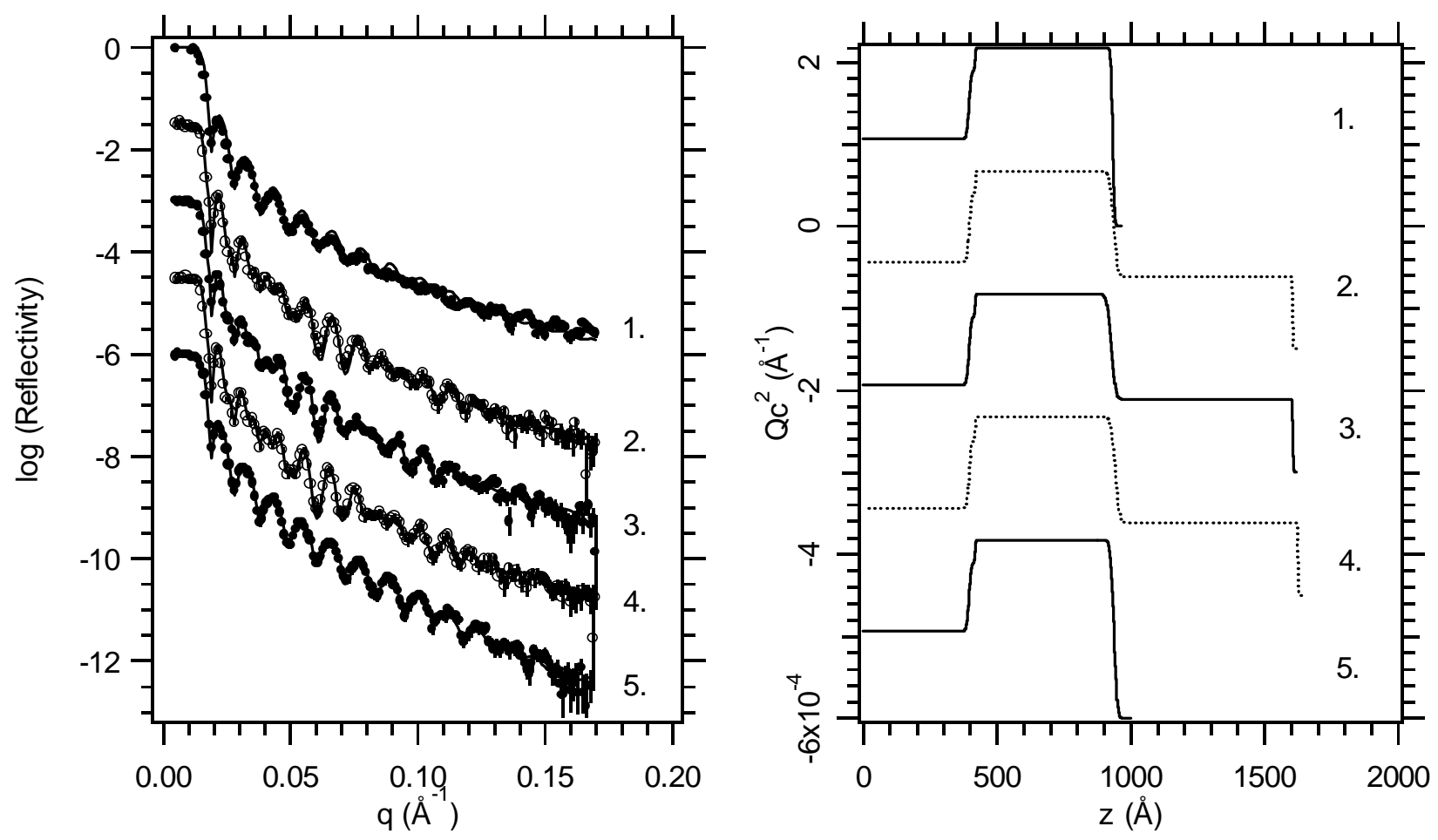

Figure 2. Neutron reflectivity data and best fits (left) and real space profiles (right) from a bilayer samples without PFOS after different processing steps: 1) the lower d-PBOCSt layer spin-coated from PGMEA 2) the bilayer after spin-coating the upper PHOSt layer (without PFOS) from 1-butanol, 3) after UV exposure with a dose of $\left.1000 \mathrm{~mJ} / \mathrm{cm}^{2}, 4\right)$ after a PEB for $90 \mathrm{~s}$ at $110^{\circ} \mathrm{C}$, and 5) after development in $0.26 \mathrm{~N}$ TMAH solution. 


\section{RESULTS AND DISCUSSION}

A control experiment for the bilayer system was performed using a PHOSt layer without PFOS to ensure that polymer interdiffusion is not of concern over the time scales and processing steps used in this study. In Figure 2a, we show the NR data and best fits for the bilayer system without PFOS after each processing step. Figure $2 b$ shows the real space profiles corresponding to the best fits. The processing steps include 1) the initial spin-coated PBOCSt layer after $\mathrm{PAB} ; 2$ ) the bilayer after the subsequent spin-coating of the PHOSt layer and another PAB; 3 ) exposure to UV; 4) a PEB of $90 \mathrm{~s}$ at $110^{\circ} \mathrm{C}$; and 5) development in $0.26 \mathrm{~N} \mathrm{TMAH}$. The reflectivity data are characterized by oscillations that arise from the constructive and destructive interference of neutrons reflected from interfaces in the bilayer stack. The periodicity, amplitude, and decay rate of the reflectivity provide sensitive measures for quantities such as the layer thickness and interfacial widths. The thickness of the initial PBOCSt layer was $(514 \pm 1) \AA$ and the surface roughness was $(12 \pm 2) \AA$. The PHOSt layer was $(669 \pm 1) \AA$ and the interfacial width between PBOCSt and PHOSt was $(29 \pm 3) \AA$. After both the UV dose and the PEB, there were no changes in the interfacial width. After development in $0.26 \mathrm{~N}$ TMAH, the remaining PBOCSt film was $(519 \pm 1) \AA$ with a surface roughness of $(23 \pm 3) \AA$. These results show that the thermally activated deprotection at the PHOSt / PBOCSt interface without the acid was not significant.
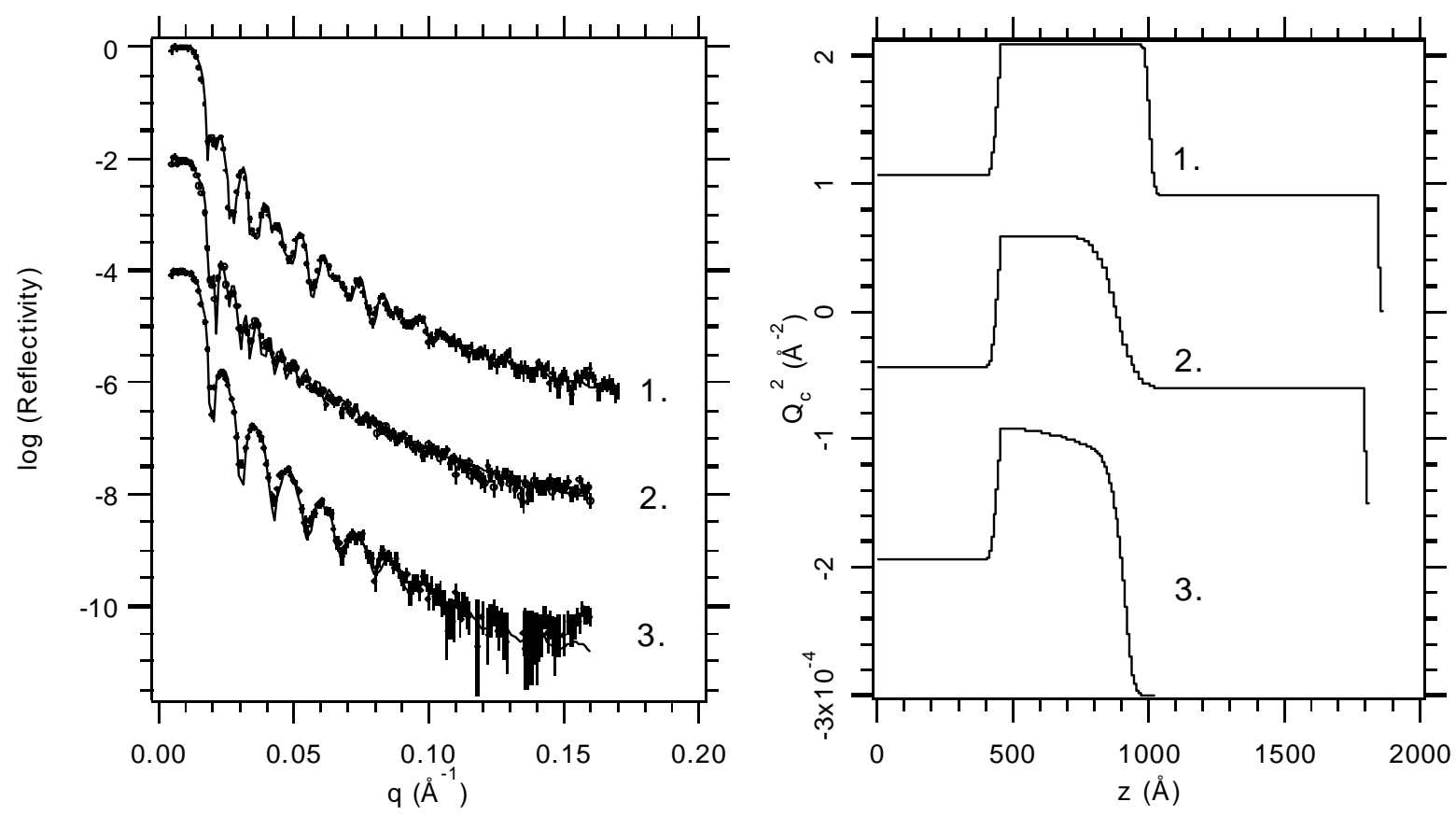

Figure 3. a) Neutron reflectivity data and best fits for bilayer samples with PFOS in the PHOSt layer after: 1) spin-coating and PAB of both layers; 2) UV exposure and PEB for $30 \mathrm{~s}$ at $110^{\circ} \mathrm{C}$ and 3) development in $0.26 \mathrm{~N}$ TMAH. b) Real space profiles corresponding to the best fits to the reflectivity data in terms of $\mathrm{Q}_{\mathrm{c}}{ }^{2}$. The abscissa origin is located within the silicon substrate and subsequently moves into the PBOCSt layer, the PHOST/PFOS layer, and the free surface.

In Figure 3a, we show the NR data along with best fits for the initial interface, after a PEB of $30 \mathrm{~s}$ at $110{ }^{\circ} \mathrm{C}$, and after development in $0.26 \mathrm{~N} \mathrm{TMAH.} \mathrm{Figure} 3 \mathrm{~b}$ shows the real space profiles corresponding to the best fits to the NR data. For the sample before PEB (after PAB), the thickness of the initial PBOCSt layer was $(544 \pm 1) \AA$, the thickness of the PHOS/PFOS layer was $(829 \pm 1) \AA$, and the interfacial width between the two layers was $(28 \pm 3) \AA$. After the PEB, the NR curve changes dramatically due to the propagation of the reaction front into the PBOCSt layer. From the real space profile, the reaction front begins at the initial interface and is broader than the initial interfacial width. The width of the error function used to fit the reaction front profile was $(139 \pm 5) \AA$. After the development step, the PHOSt/PFOS 
layer is removed along with the soluble reaction products. Here, the data from the developed bilayer and the PEB step are obtained from the same sample providing a direct correlation between the deprotection level and the final structure. The NR data after development cannot be fit with an error function profile. A more complicated profile was generated without a specific functional form.
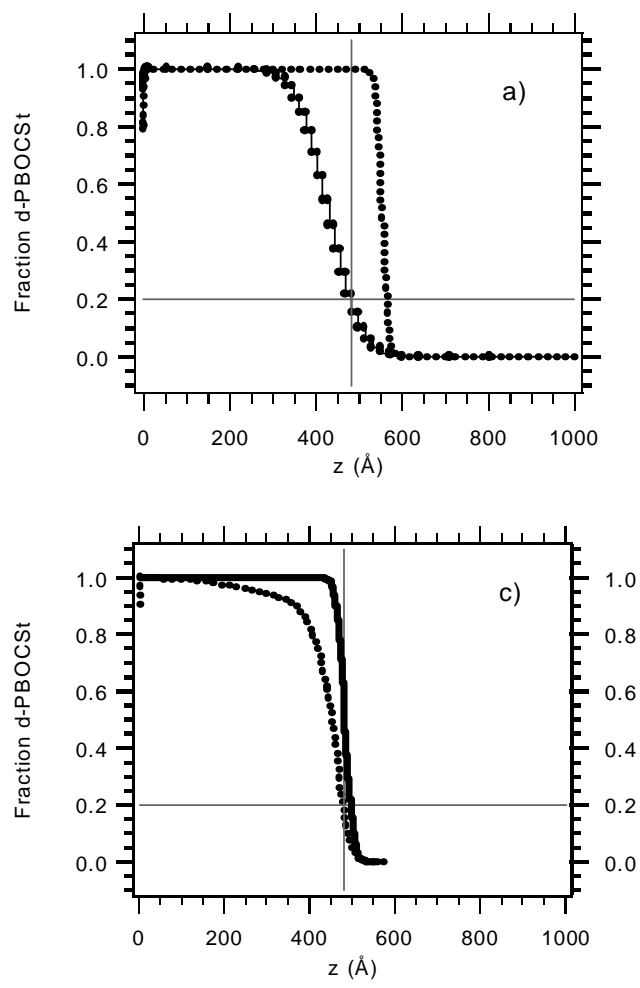
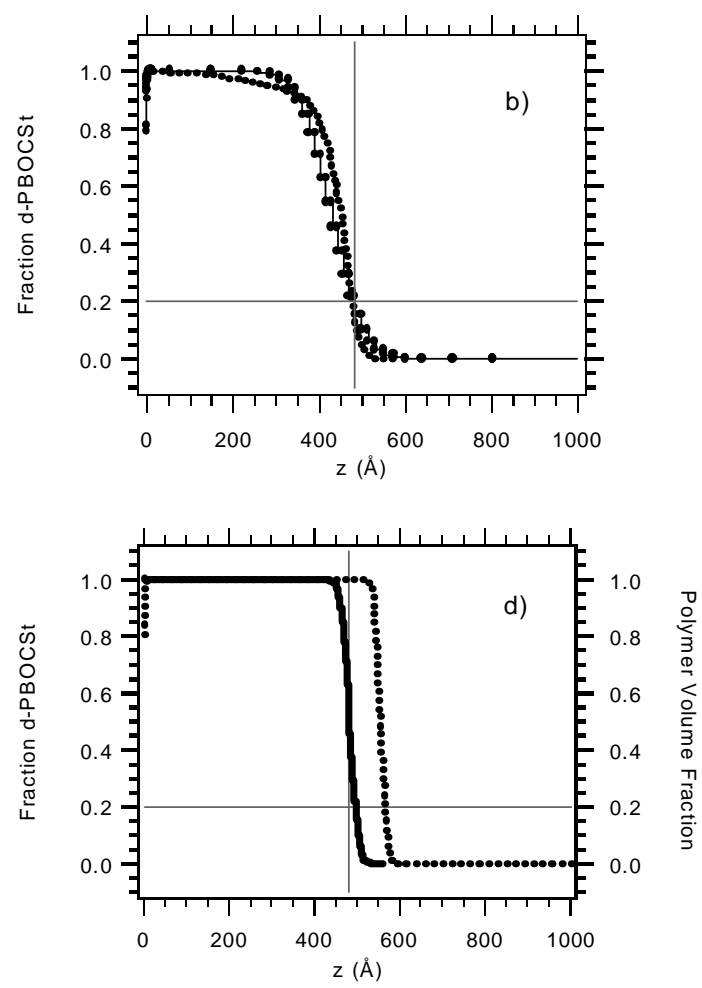

Figure 4. The real space profiles from best fits to selected neutron and $x$-ray reflectivity data normalized to the either PBOCSt protection fraction (NR) or polymer volume fraction (XR). Each graph directly compares the profiles between different processing steps. The horizontal line in each graph corresponds to the $80 \%$ deprotection level accepted as the transition point for solubility in $0.26 \mathrm{~N} \mathrm{TMAH}$. The vertical line corresponds to the thickness of the developed film determined from the midpoint of the film/air interface. The graphs compare a) the initial PBOCSt/(PHOSt/PFOS) interface (dotted line) and the buried reaction front after PEB (lines and points); b) the buried reaction front and the compositional profile after development (dotted line); c) the compositional profile and the overall volume fraction after development (solid line); and d) the initial bilayer interface and the polymer volume fraction after development.

Figure 4 shows the real space profiles from the NR and XR measurements normalized to either the remaining fraction of d-PBOCSt and the overall material volume fraction. By comparing the spatial evolution of the fractional deprotection as a function of processing history, this methodology directly tracks compositional and density changes in the sample as a function of processing history. Each figure also shows a horizontal line corresponding to the $80 \%$ deprotection fraction accepted as the composition at which the polymer becomes soluble in the developer and a vertical line corresponding to the film thickness of the developed structure determined from XR. Figure 4a shows a comparison of the initial interface and the reaction front after a $30 \mathrm{~s} \mathrm{PEB}$ at $110^{\circ} \mathrm{C}$. The reaction front is broader than the initial interface showing that the reaction front does not propagate as a sharp band defined by the initial interface. Also, the reaction front clearly starts from the initial interface position. Nearly full deprotection is observed at the initial interface serving as the acid source and subsequently propagates into the PBOCSt layer. Figure $4 \mathrm{~b}$ shows a comparison between the reaction front after PEB and the compositional profile after development in $0.26 \mathrm{~N}$ TMAH for $30 \mathrm{~s}$. The data show that the compositional profile changes slightly with development. It is possible that the developer provides sufficient 
mobility for the rearrangement of the polymer layer. For this PEB time, the final film thickness or CD corresponds well with the $80 \%$ deprotection level accepted as the solubility composition for the deprotected polymer. This data validates past experimental work while providing additional detail in the subtle changes in the compositional profile after development. However, experiments with varying PEB time show that the $80 \%$ deprotection rule does not consistently predict the correct final film thickness. The film thickness differences are nanometer is size; a level of detail unimportant for the fabrication of larger lithographic structures, but significant for the fabrication of sub-100 nm structures. Figure $4 \mathrm{c}$ compares the compositional and density profile of the developed structure. Here, the surface roughness of the developed film, $(38 \pm 3) \AA$, is narrower than the width of the compositional profile. The developed structure is more sharply defined than the reaction front showing the sensitivity of the material solubility to composition. Figure $4 \mathrm{~d}$ compares the final developed structure with the structure of the initial interface. The interfacial widths of the final structure and the initial interface are very similar. Without the reaction profile before development, one could conclude that the reaction front propagated as a sharp band defined by the width of the initial interface. However, these results definitively show that the reaction front broadens with time and the sensitivity of the solubility criteria to composition is responsible for the production of sharply defined structures for this system.

\section{SUMMARY}

We have demonstrated the complementary use of neutron and x-ray reflectometry to measure the spatial evolution of the reaction front in a chemically amplified photoresist with nanometer resolution as a function of standard photoresist processing conditions. These experiments were enabled by the synthesis of a photoresist polymer with deuterium-labeled protection groups. The deuterium provides strong contrast for neutron reflectometry without significantly changing the chemistry of the system. As a result, the neutron scattering contrast becomes proportional to the extent of deprotection. Using a bilayer geometry with a lower layer consisting of the deuterated protected polymer and an upper layer consisting of the deprotected polymer loaded with PAG, we measure compositional and density depth profiles. We show that the reaction front broadens during the PEB and is not sharply defined by the initial interface. The surface roughness of the developed structure is very similar in scale to the initial interface even though the reaction front is broad. Sharply defined structures are possible because of the sensitivity to composition of the solubility condition of the deprotected polymer to the developer. The spatial detail enabled by this methodology provides critically needed data for the development of models and processes needed for the successful control of the fabrication of sub$100 \mathrm{~nm}$ structures. By extending these studies to additional PEB conditions, PAG molecules, and materials, we aim to clearly identify the appropriate reaction-diffusion mechanisms needed to describe and model chemically amplified photoresist systems.

\section{ACKNOWLEDGEMENTS}

The authors gratefully acknowledge DARPA for financial support under contract N66001-00-C-8803. We thank Thomas Mrozek and David Medeiros for their insight and helpful discussion. JLL acknowledges support under the NRC-NIST Postdoctoral Fellowship program.

\section{REFERENCES}

1. D. L. Goldfarb, M. Angelopoulos, E. K. Lin, R. L. Jones, C. L. Soles, J. L. Lenhart, and W. L. Wu, J. Vac. Sci. Technol. B 19, 2699, 2001.

2. C. L. Soles, E. K. Lin, J. L. Lenhart, R. L. Jones, W. L. Wu, D. L. Goldfarb, and M. Angelopoulos, J. Vac. Sci. Technol. B, 19, 2690, 2001.

3. D. S. Fryer, P. F. Nealey, and J. J. de Pablo, Macromolecules 33, 6439, 2000.

4. T. H. Fedynysyn, J. W. Thackery, J. H. Georger, and M. D. Denison, J. Vac. Sci. Technol. B 12, $3888,1994$.

5. T. Itani, H. Yoshino, N. Fujimoto, and K. Kasama, J. Vac. Sci. Technol. B 14, 3026, 1995.

6. J. Nakamura, H. Ban, K. Deguchi, and A. Tanaka, Jpn. J. Appl. Phys. 30, 2619, 1991. 
7. S. V. Postnikov, M. D. Stewart, H. V. Tran, M. A. Nierode, D. R. Medeiros, T. Cao, J. Byers, S. E. Webber, and C. G. Willson, J. Vac. Sci. Technol. B 17, 3335, 1999.

8. F. A. Houle, W. D. Hinsberg, M. Morrison, M. I. Sanchez, G. Wallraff, C. Larson, and J. Hoffnagle, J. Vac. Sci. Technol. B, 18, 1874, 2000.

9. D. S. Fryer, S. Bollepali, J. J. de Pablo, and P. F. Nealey, J. Vac. Sci. Technol. B, 17, 3351, 1999.

10. M. D. Stewart, M. H. Somervell, H. V. Tran, S. V. Postnikov, and C. G. Willson, Proc. SPIE 3999, 664, 2000.

11. M. Zuniga and A. R. Neureuther, J. Vac. Sci. Technol. B 13, 2957, 1995.

12. J. S. Peterson et al., Proc. SPIE 2438, 167, 1995.

13. A. A. Krasnoperova et al., J. Vac. Sci. Technol. B 12, 3900, 1994.

14. E. Croffie, M. Cheng, and A. R. Neureuther, J. Vac. Sci. Technol. B, 17, 3339, 1999.

15. B. M. Pope, Y. Yamamoto, and D. S. Tarbell, Org. Synth., 6, 418, 1988.

16. L. G. Parratt, Phys. Rev., 95, 359, 1954.

17. J. S. Higgins and H. Benoit, Polymers and Neutron Scattering, Oxford University Press, New York, 1994.

18. G. M. Wallraff and W. D. Hinsberg, Chem. Rev. 99, 1801, 1999.

19. H. Ito and C. G. Willson, Polym. Eng. Sci. 23, 1021, 1982.

20. H. Ito and M. Sherwood, Proc. SPIE 3678, 104, 1999.

21. F. A. Houle, G. Poliskie, W. D. Hinsberg, D. Pearson, M. Sanchez, H. Ito, and J. Hoffnagle, Proc. SPIE 3999, 181, 2000.

22. S. D. Burns, M. D. Stewart, J. N. Hilfiker, R. A. Synowicki, G. M. Scmid, C. Brodsky, and C. G. Willson, Proc. $12^{\text {th }}$ Internatn'l Conf. on Photopolymers, 323, 2001. 